\title{
Anisotropic nanowire growth via a self-confined amorphous template process: A reconsideration on the role of amorphous calcium carbonate
}

\author{
Li-Bo Mao ${ }^{1}$, Lei Xue ${ }^{1}$, Denis Gebauer ${ }^{2}$, Lei Liu ${ }^{1}$, Xiao-Fang Yu ${ }^{1}$, Yang-Yi Liu ${ }^{1}$, Helmut Cölfen ${ }^{2}$, and \\ Shu-Hong $\mathrm{Yu}^{1}(\mathbb{\nabla})$ \\ ' Division of Nanomaterials and Chemistry, Hefei National Laboratory for Physical Sciences at the Microscale Collaborative Innovation \\ Center of Suzhou Nano Science and Technology, CAS Center for Excellence in Nanoscience, Department of Chemistry, University of \\ Science and Technology of China, Hefei 230026, China \\ ${ }^{2}$ Department of Chemistry, University of Konstanz, Universitätsstrasse 10, Box 714, D-78457, Konstanz, Germany
}

\section{KEYWORDS}

amorphous calcium carbonate, calcite nanowire, anisotropic growth, self-confined amorphous template

\begin{abstract}
Calcium carbonate crystals with various morphologies have been found in a variety of biospecimens and artificially synthesized structures. Usually, the diversity in morphology can be attributed to different types of interactions between the specific crystal faces and the environment or the templates used for the growth of $\mathrm{CaCO}_{3}$ crystals. On the other hand, isotropic amorphous calcium carbonate (ACC) has been recognized as the precursor of other crystalline calcium carbonate forms for both in vivo and in vitro systems. However, here we propose a self-confined amorphous template process leading to the anisotropic growth of single-crystalline calcite nanowires. Initiated by the assembly of precipitated nanoparticles, the calcite nanowires grew via the continuous precipitation of partly crystallized ACC nanodroplets onto their tips. Then, the crystalline domains in the tip, which were generated from the partly crystallized nanodroplets, coalesced in the interior of the nanowire to form a single-crystalline core. The ACC domains were left outside and spontaneously formed a protective shell to retard the precipitation of $\mathrm{CaCO}_{3}$ onto the side surface of the nanowire

and thus guided the highly anisotropic growth of nanowires as a template.
\end{abstract}

\section{Introduction}

Living creatures produce large amounts of calcium carbonate biominerals for carrying out various functions, for example, the biominerals in brittlestars function as optical micro lens arrays [1]. Most of these biogenic $\mathrm{CaCO}_{3}$ minerals are shaped into unique anisotropic morphologies exhibiting thermodynamically unstable crystal faces [1-6]. Selected area electron diffraction (SAED) and atomic force microscopy (AFM)

Address correspondence to shyu@ustc.edu.cn 
observations have revealed that some of these minerals are not single crystals but mesocrystals [7]. There has been extensive research on biomineralization to understand the design strategies adopted by organisms for fabricating these biominerals, which are very different from the minerals with abiotic origin. The synergistic effect of an insoluble template and soluble proteins has been proved to be important for biomineralization $[8,9]$. Moreover, the role of amorphous calcium carbonate (ACC) in the formation of biominerals, which has been discussed for decades, is also found to be crucial $[6,10]$. ACC is quite unstable and has the highest solubility among all forms of $\mathrm{CaCO}_{3}$. Nevertheless, this thermodynamically unstable form can be kinetically stabilized by organic molecules and inorganic ions, such as acidic proteins and magnesium ions, respectively. The stabilized/metastabilized ACC can then be readily used by organisms as the precursor for the successive crystallization in various types of biogenic $\mathrm{CaCO}_{3}$ crystals [11-13]. The relationship between the biogenic ACC and the crystallization behavior has been elucidated [14-16]. Notably, a layer of ACC and/or macromolecules exists in some specimens even when most of the ACC has crystallized [17, 18].

Inspired by biomineralization, the morphology control of synthetic $\mathrm{CaCO}_{3}$ crystals, especially those with high anisotropy, has attracted much attention $[19,20]$. Generally, the formation of anisotropic crystals can be achieved via tuning of the surface energy of crystal faces by changing the temperature and $\mathrm{pH}$, or by adding specific molecules as modifiers [21-25]. An alternative method is to use prefabricated templates with designed shapes and surface functional groups [26-29]. In short, it is necessary to have diversity either in the crystal faces or in the prefabricated templates for the anisotropic growth of crystals.

On the other hand, the emergence of amorphous phases in some reports has proved them to be important intermediates for in vitro mineralization systems [10, 30]. The stabilization and transformation of the ACC phase has been studied [31-41]. Gower et al. proposed a so-called polymer induced liquid precursor (PILP) process which has been found in many mineralization processes where polymers were used as stabilizers $[10,31]$. According to their observations, the wick effect, which was proposed for the vapor-liquid-solid process of other kinds of nanowires [42], could explain the anisotropic growth of $\mathrm{CaCO}_{3}$ nanowires [10]. Qi et al. reported the growth of calcite micropillars through the assembly of polymer-stabilized nanoparticles into pure amorphous precursor nanofibers that then crystallized into the calcite form [38]. They suggested that the anisotropic growth was induced by the dipole-dipole effect or the fusion of the nanoparticles. These theories have successfully explained many observations. However, according to our recent study, a polymer-stabilized ACC protective shell coating on the crystalline core, rather than the wick effect or specific interactions between different crystal faces and polymer molecules, is found to be crucial for the growth of highly anisotropic calcite nanowires. Moreover, this ACC shell is analogous to the ACC layers found in nacre and sea urchin spine that are the remnants of the crystallization of the biogenic ACC $[17,18]$. We thus propose a new mechanism for the ACC-induced anisotropic growth of single-crystalline calcite nanowires.

\section{Experimental}

\subsection{Preparation of Kitano solution}

$\mathrm{CO}_{2}$ gas was bubbled through a $\mathrm{CaCO}_{3}$ slurry (2 $\mathrm{g}$ of $\mathrm{CaCO}_{3}$ and $1 \mathrm{~L}$ of de-ionized water (DIW); Sangon Biotech, LLC) first at $25{ }^{\circ} \mathrm{C}$ for $6 \mathrm{~h}$, and then at $4{ }^{\circ} \mathrm{C}$ for another $15 \mathrm{~min}$ [43]. The slurry was filtered to remove the remaining $\mathrm{CaCO}_{3}$. This fresh Kitano solution was kept in a beaker sealed with a plastic wrap at $4{ }^{\circ} \mathrm{C}$ for $12 \mathrm{~h}$ for further use.

\subsection{Preparation of substrates}

Glass slides were cut into $1 \mathrm{~cm} \times 1 \mathrm{~cm}$ squares, and then treated with piranha solution (concentrated sulfuric acid: $30 \mathrm{wt} . \%$ hydrogen peroxide $=4: 1$ ) at $80{ }^{\circ} \mathrm{C}$ for $6 \mathrm{~h}$. In order to facilitate the precipitation of $\mathrm{CaCO}_{3}$ and study the growth mechanism, two types of substrates were prepared: chitin and collagen substrates. The chitin substrates were prepared by spin-coating a $2 \mathrm{wt}$ \% chitosan solution (in $2 \mathrm{wt} \%$ acetic acid; Sinopharm Chemical Reagent, LLC) onto the treated glass slides and subsequent drying of the slides at room temperature, which were then immersed 
into a mixture of methanol and acetic acid anhydride $(1: 4, v / v)$ for $6 \mathrm{~h}$ at $45^{\circ} \mathrm{C}$. These acetylated substrates were then rinsed with ethanol, 0.1 $\mathrm{M} \mathrm{NaOH}$ solution and DIW successively. For preparing the collagen substrates, $0.1 \mathrm{~g}$ of collagen (MP Biomedicals, LLC) was dispersed in $100 \mathrm{~mL}$ of $0.5 \mathrm{M}$ acetic acid. The so obtained partly dissolved suspension was applied onto the glass slides, and then dried at $40{ }^{\circ} \mathrm{C}$ for $3 \mathrm{~h}$ followed by a final wash with DIW.

\subsection{Mineralization of $\mathrm{CaCO}_{3}$}

The precipitation of $\mathrm{CaCO}_{3}$ was carried out by immersing the coated glass substrates into $50 \mathrm{~mL}$ of Kitano solution containing $\mathrm{Mg}^{2+}(2 \mathrm{mM})$ and polyacrylic acid (PAA; $0.2 \mathrm{mM}, M_{\mathrm{W}}=1,800$; Sigma-Aldrich, LLC) as additives. The beaker was then placed in a polymethyl methacrylate (PMMA) cube with twelve air holes (each $0.5 \mathrm{~mm}$ in diameter) in the lid by which the dissipation rate of $\mathrm{CO}_{2}$ could be precisely controlled (Fig. 1(a)). The temperature was maintained at $37^{\circ} \mathrm{C}$ in an incubator. In a typical experiment, four air holes were opened and the mineralization process took $48 \mathrm{~h}$. The resulting specimens were washed gently with DIW and immediately immersed in ethanol to quench the crystallization process.

\subsection{Measurements and characterization}

Scanning electron microscopy (SEM) images were obtained using a Carl Zeiss Supra 40 field emission scanning electron microscope $(5 \mathrm{kV})$. All specimens were directly observed without sputtering gold onto their surfaces. Transmission electron microscopy (TEM), high resolution TEM (HRTEM), SAED, and energy dispersive spectrometry (EDS) data were obtained using a JEOL JEM-ARM200F transmission electron microscope $(200 \mathrm{kV})$. The images were taken in a few seconds to avoid the destruction of the structures by the electron beam. TEM samples the chitin substrate was carefully dissolved in hexafluoroisopropanol, then the solvent was evaporated from this mixture and finally, the resulting precipitates were gently washed with ethanol three times. For the nanowires grown on the collagen substrates, the samples were carefully scraped away from the substrate and were then suspended in ethanol. X-ray diffraction (XRD) data were measured with a PANalytical X'Pert PRO X-ray diffractometer equipped with $\mathrm{Cu} \mathrm{K} \alpha$ radiation $(\lambda=$ $1.54056 \AA$ ). The concentrations of $\mathrm{Ca}$ and $\mathrm{Mg}$ were measured using a PerkinElmer ICP-OES Optima 7,300 DV spectrometer. The concentrations of the dissolved $\mathrm{CO}_{2}$ were measured using an FC-100 $\mathrm{CO}_{2}$ analyzer
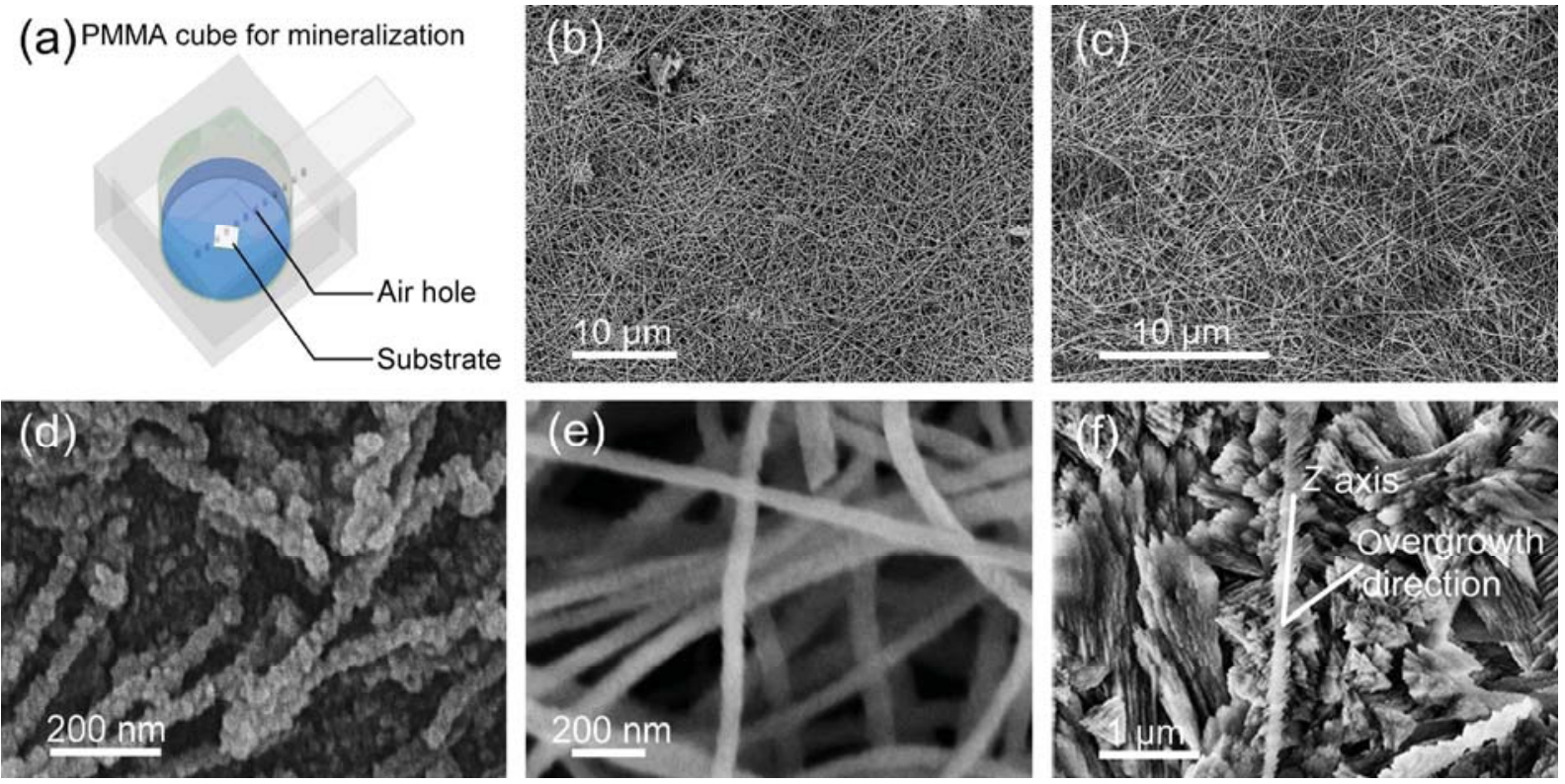

Figure 1 (a) Illustration of the experimental setup. (b) and (c) SEM images of the samples prepared in the presence of $2 \mathrm{mM} \mathrm{Mg}^{2+}$ and $0.2 \mathrm{mM}$ PAA, $\mathrm{CaCO}_{3}$ nanowires with high aspect ratio formed on both (b) chitin and (c) collagen substrates after mineralization for $48 \mathrm{~h}$. (d)-(f) Enlarged views of the specimens on chitin substrates obtained after (d) 36, (e) 48 and (f) $72 \mathrm{~h}$ of mineralization. 
(Beijing Zhecheng Tech., LLC). All pH data were collected using a Mettler Toledo FEP20 pH meter. Hydrophilic interaction liquid chromatography (HILIC) data were acquired using a Waters ACQUITY UPLC H-Class System with an ACQUITY UPLC BEH HILIC column $(1.7 \mu \mathrm{m})$. The solutions were centrifuged (at the rate of 8,000 rounds per minute for $10 \mathrm{~min}$ ) to remove larger particles and the $\mathrm{pH}$ of the solutions was adjusted to 4 by adding $0.01 \mathrm{M} \mathrm{HCl}$ before the chromatographic tests. A mixture of acetonitrile and water (5\% water for $0-4 \mathrm{~min}$, up to $80 \%$ water in $3.5 \mathrm{~min}, 30 \mathrm{~s}$ isocratic $80 \%$ water, down to $5 \%$ water in $30 \mathrm{~s}$ and reequilibriate for 5 min with $5 \%$ water) was used as the eluent. Zeta potential data were acquired using a Beckman Coulter Delta Nano C Particle Analyzer. All the specimens were tested instantly after sampling and each data point was measured 3 times.

\section{Results and discussion}

With the typical configuration, the $\mathrm{CaCO}_{3}$ nanowires were formed in the presence of $\mathrm{Mg}^{2+}$ and PAA on the substrates with widths ranging from $40-80 \mathrm{~nm}$, and lengths up to $50 \mu \mathrm{m}$ (Figs. 1(b) and 1(c); the effects of the number of open air holes and the concentrations of $\mathrm{Mg}^{2+}$ ions and PAA on the growth of $\mathrm{CaCO}_{3}$ nanowires are discussed in detail in the Electronic Supplementary Material (ESM), Fig. S1). Therefore, the formation of $\mathrm{CaCO}_{3}$ nanowires was not sensitive to the substrate type (Fig. S2 in the ESM), indicating the independence of the nanowire growth.

The growth of $\mathrm{CaCO}_{3}$ nanowires on the chitin substrate was examined by SEM to gain further insights into the mineralization process. The growth of nanowires took place in four stages: the pre-nanowiregrowth stage $(0-30 \mathrm{~h})$, the seeding stage $(30-36 \mathrm{~h})$, the booming stage (36-48 h), and the overgrowth stage $(>48 \mathrm{~h})$. Prior to the nanowire growth, with the dissipation of the dissolved $\mathrm{CO}_{2}$ (Fig. S3 in the ESM), the degree of supersaturation of $\mathrm{CaCO}_{3}$ in the solution reached a critical point and $\mathrm{CaCO}_{3}$ nanoparticles began to accumulate on the substrate from the solution to form a micrometer-thick $\mathrm{CaCO}_{3}$ particle layer that showed very weak peaks in its XRD pattern (stage 1; Figs. S4(a) and S4(e) in the ESM). Then, the aggregation and assembly of these nanoparticles initiated the formation of the nascent nanowires (stage 2; Figs. S4(b) and S4(c) in the ESM and Fig. 1(d)), which then grew along their long axes to form micrometer-long crystalline nanowires (stage 3; Fig. 1(e)). A similar mechanism has been proposed for the $\mathrm{BaSO}_{4}$ system [44]. As the reaction went on, the nanowires underwent an overgrowth on their side surfaces (stage 4; Fig. 1(f)). With an extended period of reaction time, the nanowires finally transformed to an ordered prism array structure, which exhibited a strong reflection peak in the XRD pattern attributed to the calcite $\{104\}$ planes (stage 4; Figs. S4(d) and S4(e) in the ESM).

It was observed that these nanoparticles did not aggregate and assemble to form seed-like large particles until $30 \mathrm{~h}$ after the commencement of the reaction (Fig. S4(b) in the ESM), indicating a long pre-nanowiregrowth stage. Analysis of the crystallinity of some partly crystallized ACC nanodroplets in the solution (these nanodroplets continuously precipitated onto the substrate to form the nanoparticle layer) demonstrated that almost all nanodroplets were completely amorphous up to $24 \mathrm{~h}$ (Fig. 2(a)), and began to partly crystallize only after $30 \mathrm{~h}$ from the commencement of the reaction (Figs. 2(c) and 2(d)). While the crystallinity of the nanodroplets in the solution as well as the precipitated nanoparticles increased gradually (Fig. 2 and Fig. S5 in the ESM), the initial nanowires necessarily consisted of large crystalline subunits (see below). Thus, it could be concluded that proper crystallization of the nanoparticles, was essential for the initialization of the nanowire growth. Moreover, the degrees of their crystallinity (Figs. 2(c) and 2(d)) and their sizes (Fig. 2(b)) were not quite uniform. As the crystallinity of the nanoparticles was not uniform, the nanoparticles with higher crystallinity started self-assemble earlier than the poorly crystallized particles, which led to the different growth rates of nanowires. Similarly, the non-uniformity in the nanowire diameters may be attributed to the non-uniformity in the diameters of the nanoparticles that initialize the nanowire growth.

The crystallinity of the obtained $\mathrm{CaCO}_{3}$ nanowires was confirmed by SAED (Fig. 3(a), inset). Definite diffraction spots, but not polycrystalline rings, were observed in the patterns, indicating that the nanowires had a single-crystalline nature. The single-crystallinity of the nanowires was further confirmed by the HRTEM 

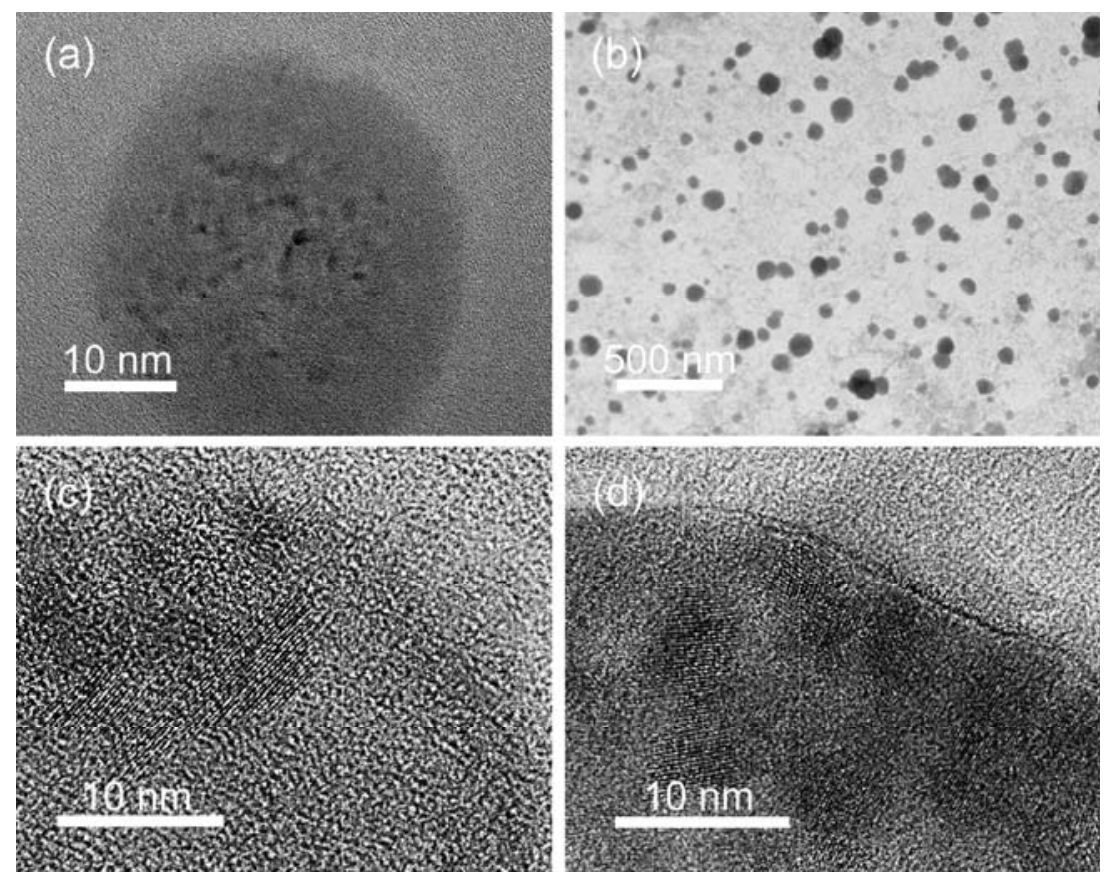

Figure 2 (a) HRTEM image of a polymer-stabilized liquid-like ACC nanodroplet (24 h, highly amorphous). The small dark regions $(<2 \mathrm{~nm}$ ) might be pre-nucleation clusters [45]. (b) TEM image of ACC nanodroplets, showing their sizes were not uniform (30 h). (c) A partly crystallized ACC nanodroplet with low crystallinity (30 h). (d) A partly crystallized ACC nanodroplet with high crystallinity (30 h).

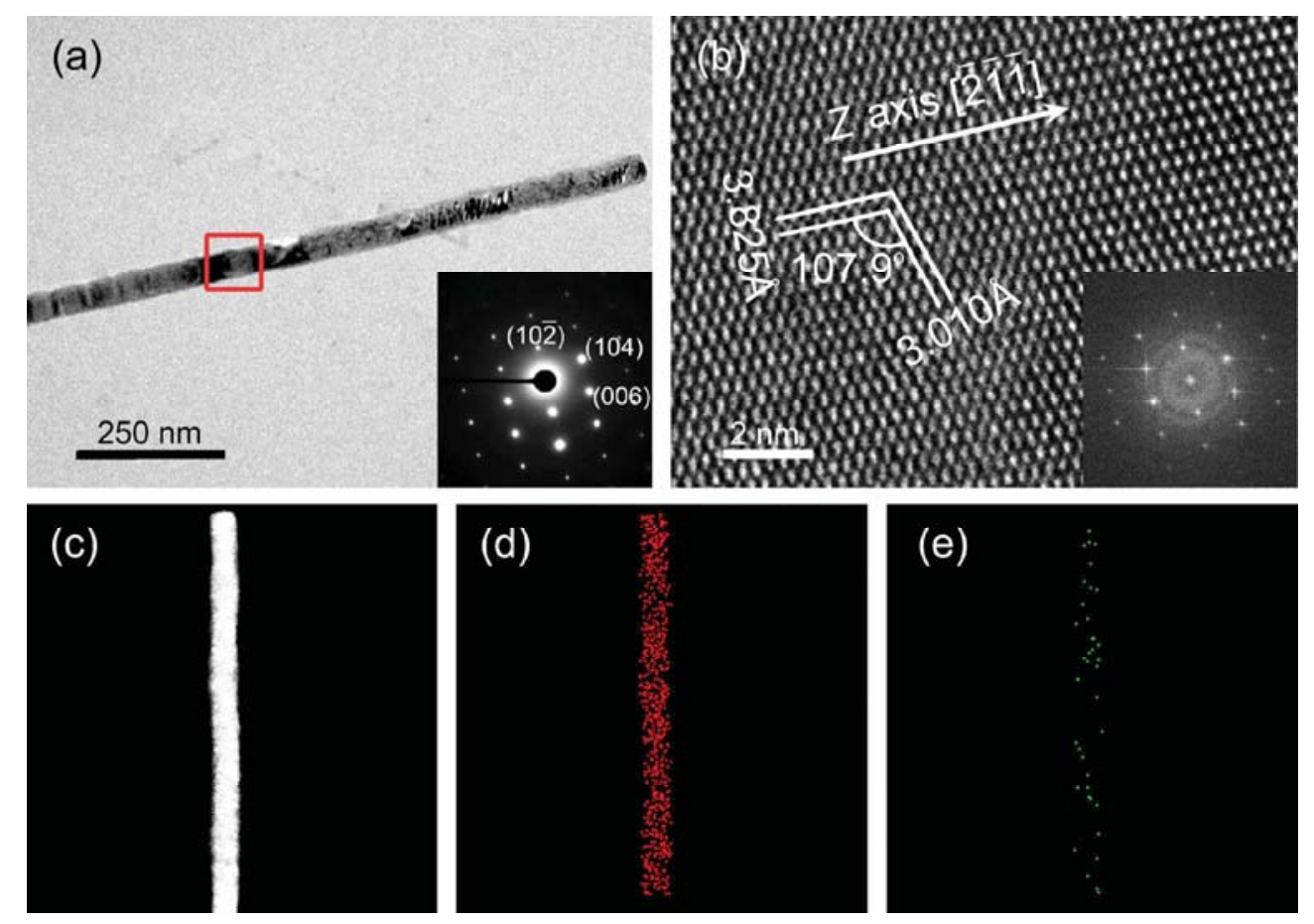

Figure 3 (a) TEM image of a $\mathrm{CaCO}_{3}$ nanowire; inset: SAED pattern. (b) HRTEM image of the red square in (a); inset: reduced fast Fourier transformation (FFT) of the HRTEM image. (c)-(e) EDS mapping of (d) Ca and (e) Mg in (c) the nanowire.

images (Fig. 3(b) and the inset). Despite the fact that the initial short nanowires were mesocrystals, no grain boundary or subunit could be observed within the long nanowires (Fig. S6 in the ESM), confirming that these nanowires were not mesocrystals, which has been defined as an assembly of small single-crystalline 
nanograins with the same mutual crystallographic orientation $[7,46]$. The fact that the nanowires were single-crystalline does not contradict their growth mechanism of their growth, because the subunits and boundaries rather than the formation mechanism are the criterion to distinguish a mesocrystal from a single crystal [46]. We examined the growth directions ( $Z$ axis) of several nanowires grown on both chitin and collagen substrates, and all of them were found to have grown in the [2111] direction (Fig. S6 in the ESM). The uniformity in their orientations was supposed to originate from the oriented assembly of $\mathrm{CaCO}_{3}$ particles during the early stages of the nanowire growth as the orientation of the initial nanowires was the same as that of the long nanowires (Fig. S7 in the ESM). According to attachment growth mechanism [21, 44], it was suggested that the PAA molecules could selectively adsorb onto and protected the specific crystal planes of these small $\mathrm{CaCO}_{3}$ particles, and then the other crystal planes with higher surface energy attached to each other to reduce the total surface energy and formed the initial nanowires, thereby providing specifically oriented seeds for the subsequent growth. Eventually, the polymer-adsorbed planes with low energy were left, e. g., $\{10 \overline{2}\}$ planes. The EDS mapping of $\mathrm{Mg}$ clearly showed a homogeneous distribution of $\mathrm{Mg}$ in the nanowires (Fig. 3(e)); the molar ratio of $\mathrm{Mg}: \mathrm{Ca}$ was measured to be 1:10.17 (Figs. 3(c)-3(e)), while the doping molar ratio of $\mathrm{Mg}: \mathrm{Ca}$ was estimated to be 1:10.64 (Fig. 3(b) and Fig. S8 in the ESM), demonstrating that most of the $\mathrm{Mg}^{2+}$ ions (ca. 95.6\%) were doped into the calcite structure.

Both $\mathrm{Mg}^{2+}$ ions and PAA are known to actively participate in the precipitation of $\mathrm{CaCO}_{3}$ as the stabilizer of ACC [10, 12, 33, 47, 48]. Moreover, ACC emerges frequently in the formation of $\mathrm{CaCO}_{3}$ fibers $[24,28,34,38,39]$. Accordingly, in immature nanowires, we found an ACC layer (Figs. 4(a)-4(d), ca. $5 \mathrm{~nm}$, marked with red borderlines; see also Figs. S9 and S10 in the ESM) coated on the crystalline interior. On the contrary, no such amorphous layer could be found on the tip area of the nanowires (Fig. 4(a) and Fig. S10(a) in the ESM). The amorphous and polycrystalline domains are fewer (Fig. 4, marked with yellow borderlines) in the areas that were farther away from the tip area, and the ACC shell became thicker and purer (Fig. 4; enlarged in Fig. S10 in the ESM). This suggested a non-classical mechanism for the growth of single crystals through the attachment and rearrangement of crystalline domains with random orientations and the
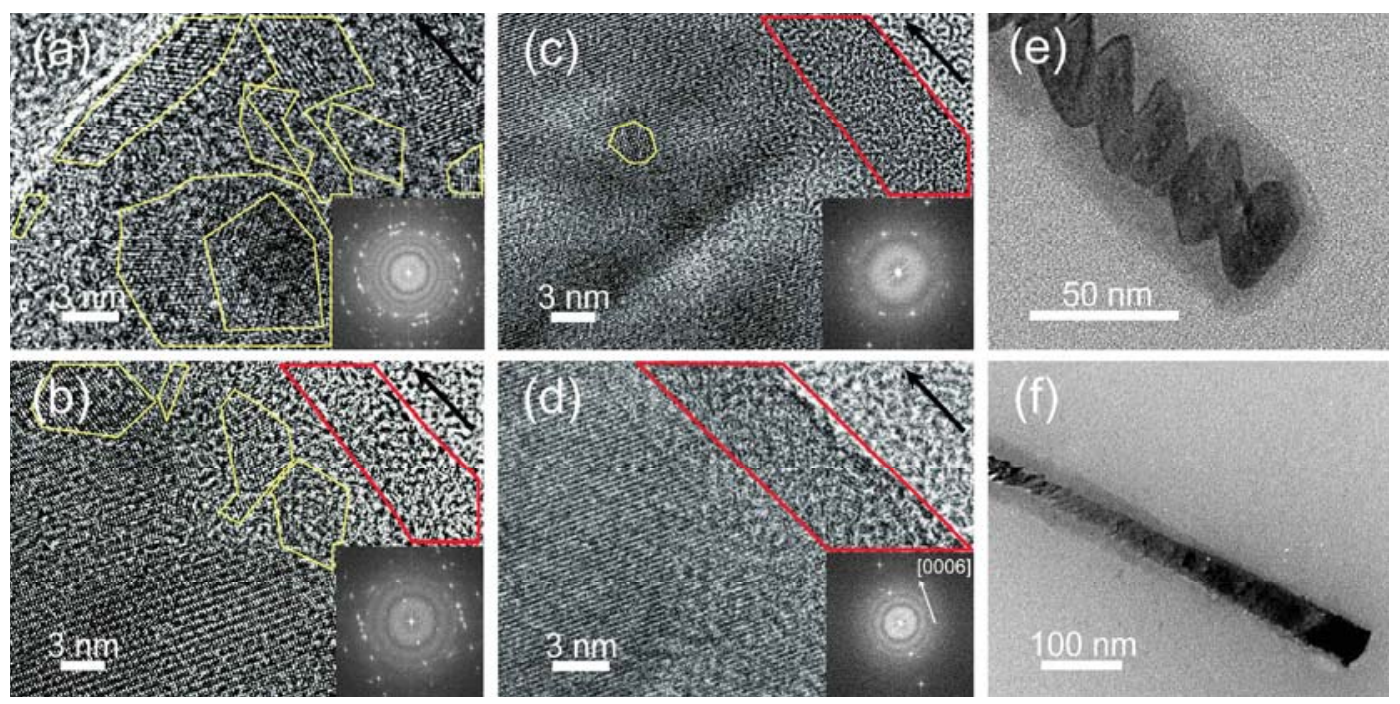

Figure 4 (a)-(d) HRTEM images of an immature nanowire $(42 \mathrm{~h})$. The black arrows in each image represent the growth direction ( $Z$ axis) of the nanowire. Its tip area is shown in (a); areas successively farther away from the tip are shown in (b)-(d), respectively. The insets are the reduced FFT patterns of the corresponding area. Regions with red borderlines are the amorphous shell layers of each area. Polycrystalline domains whose orientations are different from that of the main body of the nanowire are marked with yellow borderlines. (e) TEM image of the tip area of the initial nanowire $(36 \mathrm{~h})$ with an amorphous tip and a jagged crystalline interior. (f) A nanowire that was transforming from the slow on-the-layer growth to the fast in-the-solution growth $(36 \mathrm{~h})$. 
consequent assimilation with the single-crystalline core [19]. As a result of the aggregation and coalescence of crystalline domains to form the core, the amorphous domains were spontaneously left outside thus forming the shell [27]. However, these expelled amorphous domains did not crystallize and assimilate into the single-crystalline core instantly because they could be temporarily stabilized by the $\mathrm{Mg}^{2+}$ ions and the PAA molecules, whereby, the nanodroplets in the solution could remain partly amorphous for a long time (Fig. 2).

As we have already discussed, three mechanisms have been proposed for the anisotropic growth of $\mathrm{CaCO}_{3}$ crystals. In our experiment, any line-shaped template or crystalline seed was not used [28]. The ACC layer rather than crystal faces was exposed to the solution at the side surface of the immature nanowires and the tip surface was partly amorphous (Fig. 4(a) and Fig. S10(a) in the ESM), thus the mechanism that morphological anisotropy through the selective growth of crystal faces with different energies could be excluded. Moreover, unlike the PILP systems reported previously, we did not observe the presence of any "wick", i.e. "bobble" [31] at the tip of these $\mathrm{CaCO}_{3}$ nanowires during any of the growth stages. Thus, although this mechanism could well explain the formation of $\mathrm{CaCO}_{3}$ wires in some previous reports, it could not explain our experiments well; the experimental configurations were not the same. It might be suspected that the organic substrate played a role in the growth of the nanowires. However, it has been verified that the nanowires were developed on the top of a flat layer of $\mathrm{CaCO}_{3}$ nanoparticles and not directly on the organic substrate, and the orientations of all the nanowires were uniform for both the substrates. Therefore, the organic substrates did not directly affect the anisotropic growth. An interesting mechanism has been speculated that the accumulation of stabilizers might cause a significant difference between the side surface and the tip, which then guided the anisotropic growth of $\mathrm{CaCO}_{3}$ [39]. However, in our case, the ACC shell layer on the side surface of the nanowire rather than the stabilizers was suspected to be responsible for the highly anisotropic growth.

To understand how this shell confined and guided the growth of the crystals, the structures of the forefront of the nanowire growth and the amorphous shell were compared. Figures 4(a)-4(d) (enlarged in Fig. S10 in the ESM), show that the tip area was composed of small crystalline domains with various orientations and randomly distributed amorphous domains, and in the forefront, some crystalline surfaces were exposed to the solution. In contrast, a typical shell consisted of pure amorphous $\mathrm{CaCO}_{3}$. It was the different interactions between these two surfaces and the involved $\mathrm{CaCO}_{3}$ species that directly drove the anisotropic growth, so these species should be considered as well. It has been experimentally confirmed that in the presence of specific polymers, $\mathrm{CaCO}_{3}$ could form liquid droplets that act as precursors (i.e. PILP) for the subsequent growth [10]. In our experiments, the existence of these polymer-stabilized liquid-like ACC nanodroplets has also been directly observed and verified (Fig. 2 and Fig. S11 and Movie S1 in the ESM). The crystalline and amorphous structures observed in our experiments indicated the participation of polymer-stablizer liquidlike ACC nanodroplets as the major building blocks in the growth stage, as such complicated structure could not be well explained under the regime of classical crystallization theory [10,19]. However, although observations of the overgrowth indicated an iondominated classical pathway, the roles of other $\mathrm{CaCO}_{3}$ species such as free ions, ion pairs and small clusters [45, 49-52] before the final overgrowth stage could not be unambiguously identified based on the previous experiments and data. Nevertheless, both ions and nanoparticles have been found to be the primary units for crystallization according to a recent study on the growth of silicalite-1 [53]. Because of the much higher concentration of $\mathrm{Ca}$ (about $10.43 \mathrm{mM}$ initially and $6.35 \mathrm{mM}$ at $72 \mathrm{~h}$; Table S1 in the ESM) with respect to PAA $(0.2 \mathrm{mM}$ initially and about $0.057 \mathrm{mM}$ at 72 h; Fig. S12 in the ESM, calculated from the relative integral area) and the absence of amorphous precipitates on the nanowire tips during the overgrowth stage, the existence and participation of these $\mathrm{CaCO}_{3}$ species were quite possible with continuous and gradual dissipation of $\mathrm{CO}_{2}$ and PAA (Figs. $\mathrm{S} 3$ and $\mathrm{S} 12$ in the ESM). In other words, besides the verified ACC nanodroplets, other $\mathrm{CaCO}_{3}$ species may also precipitate on the tip of the nanowire through a competitive growth mechanism [19].

Based on the above analyses, it could be concluded 
that this ACC shell played the role of a template for the growth of $\mathrm{CaCO}_{3}$ nanowires. The ACC with higher chemical potential is thermodynamically unstable compared to the calcite form. As a result, the ACC shell subsequently crystallized and transformed into calcite (Fig. S6 in the ESM, mature nanowire). It is known that in heterogeneous nucleation process, a surface with lower chemical potential can reduce the energy required for forming a new surface and thus facilitate the nucleation better $[13,19]$. As a consequence, the ACC shell was not as good as the crystal face for the heterogeneous nucleation. Therefore, the forefront where many calcite crystal faces were exposed was more favorable for the heterogeneous nucleation. This surface selectivity driven by the free surface energy was responsible for the precipitation of both ACC nanodroplets and other possible $\mathrm{CaCO}_{3}$ species in the solution. It should be noticed that there have been reports of the ACC serving as the nuclei for the heterogeneous nucleation [52]. However, the properties of the ACC were quite different in our work. Therefore, our conclusion that the ACC shell significantly retarded the precipitation of $\mathrm{CaCO}_{3}$ did not contradict previous observations. On the other hand, the ACC nanodroplets, which were stabilized by the negatively charged PAA, had a net negative charge (ESM, Table S2). Consequently, the ACC shell derived from these ACC nanodroplets also had a negative surface charge. Therefore, there was a repulsive electrostatic force between the ACC shell and the ACC nanodroplets. It was believed that the crystallized $\mathrm{CaCO}_{3}$ contained less stabilizer molecules because the crystallization suggests that they were de-stabilized. Moreover, the crystallization-induced extrusion of the additives, which then accumulated at the interface, has been confirmed previously [26]. Consequently, the repulsive force was weaker between the tip and the ACC nanodroplets because there were many crystalline surfaces exposed to the solution in the forefront (Fig. S10(a) in the ESM). Hence, these ACC nanodroplets preferred to precipitate on the tip of the nanowires rather than the ACC shell. In addition, as we have reported previously [47], the selective wettability of the nanodroplets with different surfaces in the presence of $\mathrm{Mg}^{2+}$ ions might also contribute to the anisotropic growth. We can thus explain why a proper concentration of $\mathrm{Mg}^{2+}$ ions best facilitated the formation of nanowires (Fig. S1 in the ESM). Although the ACC shell in the nanowires can exist for more than a month in dry air or ethanol, as the reaction went on and $\mathrm{CO}_{2}$ as well as PAA continuously dissipated, it inevitably crystallized and the nanowires ripened in the solution (42 to 48 h; Figs. S6 and S10 in the ESM). On the other hand, in the absence of the ACC shell, the side surfaces of the nanowires started to grow and eventually the nanowires vanished (Fig. 1(f) and Fig. S4(d) in the ESM), which in turn confirms the template role of the ACC shell.

The self-confined amorphous template process is illustrated in Fig. 5. $\mathrm{CaCO}_{3}$ precursors in the solution: the ACC nanodroplets (green) and possibly free ions, ion pairs and, clusters (purple), approached the nanowire from various directions. However, the ACC shell (yellow) on the side surface of the nanowire prevented the precipitation of these precursors because of its higher surface energy and the stronger repulsive electrostatic force between the surface and the charged nanodroplets (red arrows). Therefore, the nanowires could only grow along their $Z$ axis. Once a liquid-like nanodroplet coalesced with the tip, it would not solidify instantly but remain liquid-like (light green). Hence, the polycrystalline domains (blue), which were created by the partly crystallized nanodroplets, were still rotation-free and assimilated by the singlecrystalline interior (steel blue) via axis rotation and lattice match, or via Ostwald ripening [54,55], and then the amorphous domains in the tip were left outside to form the new shell. As this process continued, the nanowire kept growing.

Besides the highly anisotropic growth of the nanowires, the different growth speeds in stages 2 and 3 could also be attributed to the difference between the ACC surface and the surface exposing many crystal faces. No nanowire was formed in the first $30 \mathrm{~h}$. Then, after $6 \mathrm{~h}(30-36 \mathrm{~h})$, the nanowires started to form but most of them were still very short (Fig. S4(c) in the ESM). However, in the next $12 \mathrm{~h}(36-48 \mathrm{~h})$, the length of the nanowires boomed to dozens of microns and covered the substrate (Fig. 1(b)), indicating a transition in the growth kinetics. By comparing the tips of two nanowires from these two stages, it was found that the tip area of a short nanowire $(36 \mathrm{~h})$ was totally 


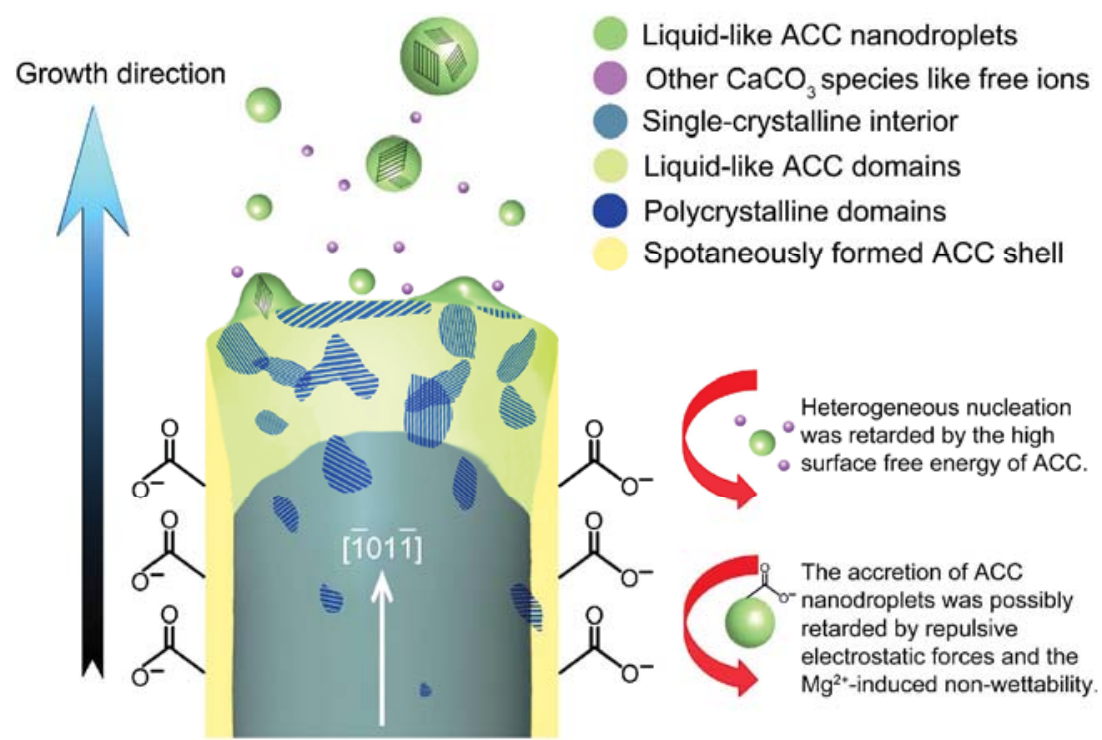

Figure 5 Schematically illustrating the proposed mechanism of nanowire growth (see the context for detailed explanation).

amorphous which was analogous to the ACC shell at the side surface (Fig. 4(e)), while the tip of an immature long nanowire $(42 \mathrm{~h}$ ) was partly crystallized (Fig. S10(a) in the ESM). The significant differences between these two surfaces gave rise to different growth kinetics. A possible explanation for this is that at the beginning of the nanowire growth, the nanoparticles that had high crystallinity and large single-crystalline domains and were close to the tips of the nascent short wires lying on the top of the precipitated $\mathrm{CaCO}_{3}$ particle layer, were able to attach to the tips crystal-face-selectively; the probability of contact and match between the particles with large single-crystalline domains and the nanowire tips was much higher than that between the particles with lower crystallinity and the nanowire tips (the crystallinity of the nanodroplets, i.e. the precursor of the nanoparticles, was not uniform; see Figs. 2(b)-2(d)). The coalescence of the poorly crystallized nanoparticles was retarded by the electrostatic force originating from the ACC layer on the nanowire tip. Despite the fact that the attachments of the nanoparticles with high crystallinity to the nanowire tip were permitted, the initial growth was slow because the contact and match took time and only a part of the nanoparticles fulfilled the condition of crystallinity. Consequently, the short nanowires were quite distinct from the long ones; the jagged crystalline interior was composed of large subunits and the tip was totally amorphous since the crystalline domains were assimilated during the slow growth period (Fig. 4(e)). This process was significantly accelerated when the nanowires grew longer, left the surface of the precipitated particle layer, and stretched into the solution. At this stage, regardless of the crystallinity, all ACC nanodroplets in the solution were able to attach to the tip of the nanowire because of their considerable kinetic energy brought about by Brownian motion. These new participants changed the tip of the nanowire into a complicated one where some crystalline surfaces were exposed to the solution (Fig. S10(a) in the ESM) and in turn, further weakened the electrostatic barrier and facilitated the attachment of new nanodroplets. As a matter of fact, the intermediate state of these two stages has already been captured (Fig. 4(f)). Both the morphology change of the crystalline interior and the absence of an amorphous tip clearly illustrated this transformation. Notably, the dark tip did not mean the crystallinity was higher than the jagged part, but rather indicated a tip consisting of many polycrystalline and amorphous domains.

\section{Conclusions}

In summary, we propose a new mechanism by which the polymer-stabilized ACC shell could guide the 
anisotropic growth of single-crystalline calcite nanowires through a self-confined amorphous template process. The amorphous template prevented the overgrowth of the nanowires on their side surfaces by retarding the precipitation of $\mathrm{CaCO}_{3}$ via liquidlike precursor-mediated as well as ion-by-ion growth pathways. By implementing the self-confined amorphous template process in artificial environments, it can be better understood how the living organisms carry out biomineralization and control the various aspects of biominerals, such as their shapes and radii. Moreover, the present work may shed light on the understanding of in vitro biomineralization and help to develop new strategies for the synthesis of other crystals via amorphous precursors.

\section{Acknowledgements}

This work was funded by the National Natural Science Foundation of China (Nos. 21521001, 21431006, 21061160492, and J1030412), the National Basic Research Program of China (Nos. 2014CB931800 and 2013CB933900), the Users with Excellence and Scientific Research Grant of Hefei Science Center of CAS (Nos. 2015HSC-UE007 and 2015SRG-HSC038), and the Chinese Academy of Sciences (No. KJZD-EW-M01-1).

\section{References}

[1] Aizenberg, J.; Tkachenko, A.; Weiner, S.; Addadi, L.; Hendler, G. Calcitic microlenses as part of the photoreceptor system in brittlestars. Nature 2001, 412, 819-822.

[2] Huang, Z. W.; Li, X. D. Origin of flaw-tolerance in nacre. Sci. Rep. 2013, 3, 1693.

[3] Zimmermann, E. A.; Gludovatz, B.; Schaible, E.; Dave, N. K. N.; Yang, W.; Meyers, M. A.; Ritchie, R. O. Mechanical adaptability of the Bouligand-type structure in natural dermal armour. Nat. Commun. 2013, 4, 2634.

[4] Launey, M. E.; Buehler, M. J.; Ritchie, R. O. On the mechanistic origins of toughness in bone. Ann. Rev. Mater. Res. 2010, 40, 25-53.
[5] Gordon, L. M.; Cohen, M. J.; MacRenaris, K. W.; Pasteris, J. D.; Seda, T.; Joester, D. Amorphous intergranular phases control the properties of rodent tooth enamel. Science $\mathbf{2 0 1 5}$, 347, 746-750.

[6] Politi, Y.; Arad, T.; Klein, E.; Weiner, S.; Addadi, L. Sea urchin spine calcite forms via a transient amorphous calcium carbonate phase. Science 2004, 306, 1161-1164.

[7] Li, X. D.; Xu, Z.-H.; Wang, R. Z. In situ observation of nanograin rotation and deformation in nacre. Nano Lett. 2006, 6, 2301-2304.

[8] Fritz, M.; Belcher, A. M.; Radmacher, M.; Walters, D. A.; Hansma, P. K.; Stucky, G. D.; Morse, D. E.; Mann, S. Flat pearls from biofabrication of organized composites on inorganic substrates. Nature 1994, 371, 49-51.

[9] Yao, H.-B.; Ge, J.; Mao, L.-B.; Yan, Y.-X.; Yu, S.-H. 25th anniversary article: Artificial carbonate nanocrystals and layered structural nanocomposites inspired by nacre: Synthesis, fabrication and applications. Adv. Mater. 2014, 26, 163-188.

[10] Gower, L. B. Biomimetic model systems for investigating the amorphous precursor pathway and its role in biomineralization. Chem. Rev. 2008, 108, 4551-4627.

[11] Addadi, L.; Raz, S.; Weiner, S. Taking advantage of disorder: Amorphous calcium carbonate and its roles in biomineralization. Adv. Mater. 2003, 15, 959-970.

[12] Aizenberg, J.; Lambert, G.; Weiner, S.; Addadi, L. Factors involved in the formation of amorphous and crystalline calcium carbonate: A study of an ascidian skeleton. J. Am. Chem. Soc. 2002, 124, 32-39.

[13] Sommerdijk, N. A. J. M.; de With, G. Biomimetic $\mathrm{CaCO}_{3}$ mineralization using designer molecules and interfaces. Chem. Rev. 2008, 108, 4499-4550.

[14] Politi, Y.; Metzler, R. A.; Abrecht, M.; Gilbert, B.; Wilt, F. H.; Sagi, I.; Addadi, L.; Weiner, S.; Gilbert, P. U. P. A. Transformation mechanism of amorphous calcium carbonate into calcite in the sea urchin larval spicule. Proc. Natl. Acad. Sci. USA 2008, 105, 17362-17366.

[15] Radha, A. V.; Forbes, T. Z.; Killian, C. E.; Gilbert, P. U. P. A.; Navrotsky, A. Transformation and crystallization energetics of synthetic and biogenic amorphous calcium carbonate. Proc. Natl. Acad. Sci. USA 2010, 107, 16438-16443.

[16] Gong, Y. U. T.; Killian, C. E.; Olson, I. C.; Appathurai, N. P.; Amasino, A. L.; Martin, M. C.; Holt, L. J.; Wilt, F. H.; Gilbert, P. U. P. A. Phase transitions in biogenic amorphous calcium carbonate. Proc. Natl. Acad. Sci. USA 2012, 109, 6088-6093.

[17] Nassif, N.; Pinna, N.; Gehrke, N.; Antonietti, M.; Jäger, C.; Cölfen, H. Amorphous layer around aragonite platelets in nacre. Proc. Natl. Acad. Sci. USA 2005, 102, 12653-12655.

[18] Seto, J.; Ma, Y. R.; Davis, S. A.; Meldrum, F.; Gourrier, A.; 
Kim, Y.-Y.; Schilde, U.; Sztucki, M.; Burghammer, M.; Maltsev, S. et al. Structure-property relationships of a biological mesocrystal in the adult sea urchin spine. Proc. Natl. Acad. Sci. USA 2012, 109, 3699-3704.

[19] Meldrum, F. C.; Cölfen, H. Controlling mineral morphologies and structures in biological and synthetic systems. Chem. Rev. 2008, 108, 4332-4432.

[20] Dey, A.; de With, G.; Sommerdijk, N. A. J. M. In situ techniques in biomimetic mineralization studies of calcium carbonate. Chem. Soc. Rev. 2010, 39, 397-409.

[21] Yu, S. H.; Cölfen, H.; Tauer, K.; Antonietti, M. Tectonic arrangement of $\mathrm{BaCO}_{3}$ nanocrystals into helices induced by a racemic block copolymer. Nat. Mater. 2005, 4, 51-55.

[22] Zhang, Q.; Liu, S.-J.; Yu, S.-H. Recent advances in oriented attachment growth and synthesis of functional materials: Concept, evidence, mechanism, and future. J. Mater. Chem. 2009, 19, 191-207.

[23] Chen, S.-F.; Zhu, J.-H.; Jiang, J.; Cai, G.-B.; Yu, S.-H. Polymer-controlled crystallization of unique mineral superstructures. Adv. Mater. 2010, 22, 540-545.

[24] Natalio, F.; Corrales, T. P.; Panthöfer, M.; Schollmeyer, D.; Lieberwirth, I.; Müller, W. E. G.; Kappl, M.; Butt, H.-J.; Tremel, W. Flexible minerals: Self-assembled calcite spicules with extreme bending strength. Science 2013, 339, 1298-1302.

[25] Noorduin, W. L.; Grinthal, A.; Mahadevan, L.; Aizenberg, J. Rationally designed complex, hierarchical microarchitectures. Science 2013, 340, 832-837.

[26] Aizenberg, J.; Müller, D. A.; Grazul, J. L.; Hamann, D. R. Direct fabrication of large micropatterned single crystals. Science 2003, 299, 1205-1208.

[27] Li, C.; Qi, L. M. Bioinspired fabrication of 3D ordered macroporous single crystals of calcite from a transient amorphous phase. Angew. Chem., Int. Ed. 2008, 47, 23882393.

[28] Kim, Y.-Y.; Hetherington, N. B. J.; Noel, E. H.; Kröger, R.; Charnock, J. M.; Christenson, H. K.; Meldrum, F. C. Capillarity creates single-crystal calcite nanowires from amorphous calcium carbonate. Angew. Chem., Int. Ed. 2011, 50, 12572-12577.

[29] Sakamoto, T.; Nishimura, Y.; Nishimura, T.; Kato, T. Photoimaging of self-organized $\mathrm{CaCO}_{3} /$ polymer hybrid films by formation of regular relief and flat surface morphologies. Angew. Chem., Int. Ed. 2011, 50, 5856-5859.

[30] Cusack, M.; Freer, A. Biomineralization: Elemental and organic influence in carbonate systems. Chem. Rev. 2008, 108, 4433-4454.

[31] Olszta, M. J.; Gajjeraman, S.; Kaufman, M.; Gower, L. B. Nanofibrous calcite synthesized via a solution-precursorsolid mechanism. Chem. Mater. 2004, 16, 2355-2362.
[32] Volkmer, D.; Harms, M.; Gower, L.; Ziegler, A. Morphosynthesis of nacre-type laminated $\mathrm{CaCO}_{3}$ thin films and coatings. Angew. Chem., Int. Ed. 2005, 44, 639-644.

[33] Oaki, Y.; Kajiyama, S.; Nishimura, T.; Imai, H.; Kato, T. Nanosegregated amorphous composites of calcium carbonate and an organic polymer. Adv. Mater. 2008, 20, 3633-3637.

[34] Kim, Y.-Y.; Kulak, A. N.; Li, Y. T.; Batten, T.; Kuball, M.; Armes, S. P.; Meldrum, F. C. Substrate-directed formation of calcium carbonate fibres. J. Mater. Chem. 2009, 19, 387-398.

[35] Homeijer, S. J.; Barrett, R. A.; Gower, L. B. Polymer-induced liquid-precursor (PILP) process in the non-calcium based systems of barium and strontium carbonate. Cryst. Growth Des. 2010, 10, 1040-1052.

[36] Wolf, S. E.; Leiterer, J.; Pipich, V.; Barrea, R.; Emmerling, F.; Tremel, W. Strong stabilization of amorphous calcium carbonate emulsion by ovalbumin: Gaining insight into the mechanism of "polymer-induced liquid precursor" processes. J. Am. Chem. Soc. 2011, 133, 12642-12649.

[37] Lee, K.; Wagermaier, W.; Masic, A.; Kommareddy, K. P.; Bennet, M.; Manjubala, I.; Lee, S.-W.; Park, S. B.; Cölfen, H.; Fratzl, P. Self-assembly of amorphous calcium carbonate microlens arrays. Nat. Commun. 2012, 3, 725.

[38] Long, X.; Ma, Y. R.; Cho, K. R.; Li, D. S.; De Yoreo, J. J.; Qi, L. M. Oriented calcite micropillars and prisms formed through aggregation and recrystallization of poly(acrylic acid) stabilized nanoparticles. Cryst. Growth Des. 2013, 13, 3856-3863.

[39] Cantaert, B.; Verch, A.; Kim, Y.-Y.; Ludwig, H.; Paunov, V. N.; Kröger, R.; Meldrum, F. C. Formation and structure of calcium carbonate thin films and nanofibers precipitated in the presence of poly(allylamine hydrochloride) and magnesium ions. Chem. Mater. 2013, 25, 4994-5003.

[40] Ihli, J.; Wong, W. C.; Noel, E. H.; Kim, Y.-Y.; Kulak, A. N.; Christenson, H. K.; Duer, M. J.; Meldrum, F. C. Dehydration and crystallization of amorphous calcium carbonate in solution and in air. Nat. Commun. 2014, 5, 3169.

[41] Smeets, P. J. M.; Cho, K. R.; Kempen, R. G. E.; Sommerdijk, N. A. J. M.; De Yoreo, J. J. Calcium carbonate nucleation driven by ion binding in a biomimetic matrix revealed by in situ electron microscopy. Nat. Mater. 2015, 14, 394-399.

[42] Laocharoensuk, R.; Palaniappan, K.; Smith, N. A.; Dickerson, R. M.; Werder, D. J.; Baldwin, J. K.; Hollingsworth, J. A. Flow-based solution-liquid-solid nanowire synthesis. Nat. Nanotechnol. 2013, 8, 660-666.

[43] Kitano, Y. A study of the polymorphic formation of calcium carbonate in thermal springs with an emphasis on the effect of temperature. Bull. Chem. Soc. Jpn. 1962, 35, 1980-1985. 
[44] Wang, T.; Reinecke, A.; Cölfen, H. In situ investigation of complex $\mathrm{BaSO}_{4}$ fiber generation in the presence of sodium polyacrylate. 2. Crystallization mechanisms. Langmuir 2006, 22, 8986-8994.

[45] Pouget, E. M.; Bomans, P. H. H.; Goos, J. A. C. M.; Frederik, P. M.; de With, G.; Sommerdijk, N. A. J. M. The initial stages of template-controlled $\mathrm{CaCO}_{3}$ formation revealed by cryo-TEM. Science 2009, 323, 1455-1458.

[46] Song, R. Q.; Cölfen, H. Mesocrystals-Ordered nanoparticle superstructures. Adv. Mater. 2010, 22, 1301-1330.

[47] Cheng, X. G.; Varona, P. L.; Olszta, M. J.; Gower, L. B. Biomimetic synthesis of calcite films by a polymer-induced liquid-precursor (PILP) process. J. Cryst. Growth 2007, 307, 395-404.

[48] Berg, J. K.; Jordan, T.; Binder, Y.; Börner, H. G.; Gebauer, D. $\mathrm{Mg}^{2+}$ tunes the wettability of liquid precursors of $\mathrm{CaCO}_{3}$ : Toward controlling mineralization sites in hybrid materials. J. Am. Chem. Soc. 2013, 135, 12512-12515.

[49] Gebauer, D.; Völkel, A.; Cölfen, H. Stable prenucleation calcium carbonate clusters. Science 2008, 322, 1819-1822.
[50] Kellermeier, M.; Picker, A.; Kempter, A.; Cölfen, H.; Gebauer, D. A straightforward treatment of activity in aqueous $\mathrm{CaCO}_{3}$ solutions and the consequences for nucleation theory. Adv. Mater. 2014, 26, 752-757.

[51] Gebauer, D.; Kellermeier, M.; Gale, J. D.; Bergström, L.; Cölfen, H. Pre-nucleation clusters as solute precursors in crystallisation. Chem. Soc. Rev. 2014, 43, 2348-2371.

[52] Nielsen, M. H.; Aloni, S.; De Yoreo, J. J. In situ TEM imaging of $\mathrm{CaCO}_{3}$ nucleation reveals coexistence of direct and indirect pathways. Science 2014, 345, 1158-1162.

[53] Lupulescu, A. I.; Rimer, J. D. In situ imaging of silicalite-1 surface growth reveals the mechanism of crystallization. Science 2014, 344, 729-732.

[54] Li, X. D.; Huang, Z. W. Unveiling the formation mechanism of pseudo-single-crystal aragonite platelets in nacre. Phys. Rev. Lett. 2009, 102, 075502.

[55] Li, D.; Nielsen, M. H.; Lee, J. R. I.; Frandsen, C.; Banfield, J. F.; De Yoreo, J. J. Direction-specific interactions control crystal growth by oriented attachment. Science 2012, 336, 1014-1018 